\title{
Applications of Graphene Liquid Cell
}

\author{
Lopa Bhatt, Nathan Rosenmann, Jinglong Guo, Mark Schlossman and Robert Klie \\ University of Illinois at Chicago, Chicago, Illinois, United States
}

One major drawback for high-resolution characterization in a transmission electron microscope remains the requirement for high vacuum in the sample region, which has long prevented us from examining liquid samples. Recently, major efforts have gone into the development of membrane-based liquid cell stages, where the liquid is separated from the vacuum in the column by a $\mathrm{SiN}_{\mathrm{X}}$ membrane as thin as $25 \mathrm{~nm}$. However, such window layers reduce the attainable spatial resolution and chemical sensitivity of electron energy-loss (EELS) or energy dispersive X-ray spectroscopies. Over the last several years, efforts to further reduce the window-layer thickness, and improve the spatial resolution of liquid cell TEM, have resulted in the successful fabrication of graphene or boron nitride liquid cells, consisting of a few layers of 2-dimensional materials encapsulating the liquid sample.[1,2,5] These advances have led to applications of liquid cells electron microscopy in many different fields, such as catalysis, materials synthesis or bio-medical imaging. [6,3]

The focus of the work presented here is to explore some interesting applications of graphene liquid cell (GLC), including the study of nanoparticles (NPs) in solution. GLCs are used to obtain high-resolution images of nanoparticles while in different solutions, and EELS is used to determine the chemical composition or oxidation states of those NPs. Our liquid cells are fabricated using 3-5 single layers of Graphene to encapsulate the nanoparticle solution as shown in Figure 1. In addition to this approach, we will also examine alternative preparation methods, using, for example, a humidifier to deposit the solution between the two layers of graphene, in an effort to increase the success rate of the liquid cell preparation process.

GLC has also been used to understand the ordering of charged nanoparticles in nanoconfined space. This project has applications in water purification system and cancer treatments.[4] The focus of this work is to examine charge screening of positive and negatively charged gold nanoparticles ( $+/-$ AuNP) in water with different concentrations of salt $(\mathrm{NaCl})$. The degree of aggregation of the charged NPs is determined by measuring the radial distribution function (RDF) of a field of particles as shown in Figure 2(b). The $\mathrm{RDF}$ is a measure of the interparticle potential and is used to compare images with high numbers of particles to examine how the distribution of particles differs with concentration of $\mathrm{NaCl}$. This information yields a much better understanding of the aggregation behavior of charged Au NP in water.

Oral biology is another field of study were GLCs can be used to, for example, explore the structure and composition of biofilm formation. In the project presented here, GLCs are used to characterize the effects of ceria $\left(\mathrm{CeO}_{2}\right)$ nanoparticles in the formation of oral biofilms. GLCs are used to identify the effects of particle size, crystallinity and surface modification on the agglomeration of $\mathrm{CeO} 2$ nanoparticles. Atomicresolution high-angle annular dark-field (HAADF) imaging in a scanning transmission electron microscope (STEM) is used to characterize the $\mathrm{CeO}_{2}$ nanoparticles in solution with different $\mathrm{pH}$, while EELS of the Ce M-edges can determine the Ce valence state as shown in Figure 2(a).[3,7] 
1.

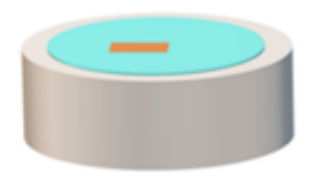

Graphene on copper substrate in etchant solution

4.

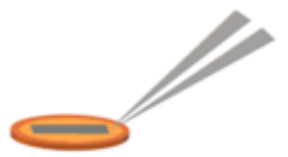

Transferring Graphene film from deionized water to TEM grid
2.

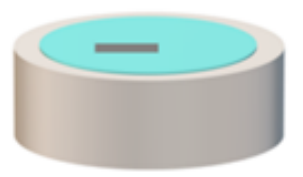

Monolayer of Graphene floating on the etchant solution

5.

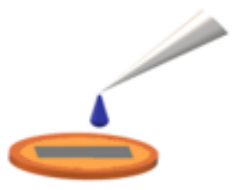

Depositing solution one wants to examine onto Graphene coated grid
3.
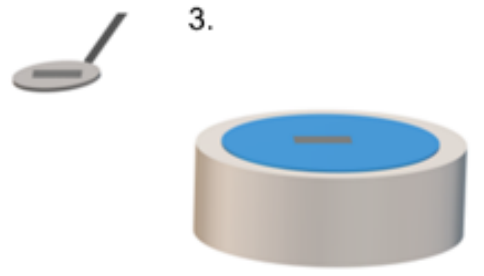

Transfer of Graphene film from etchant solution to deionized water

6.

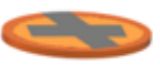

Transferring second layer of Graphene onto the grid

Figure 1. Procedure of making Liquid Cell

a.

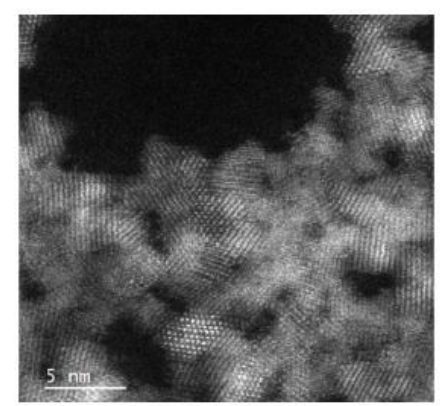

b.

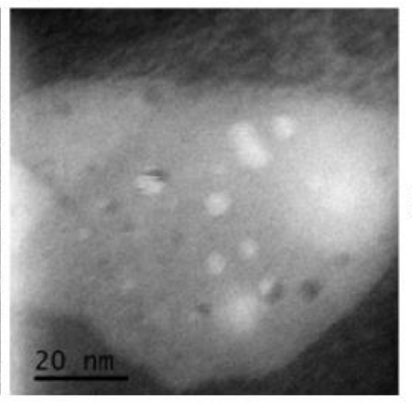

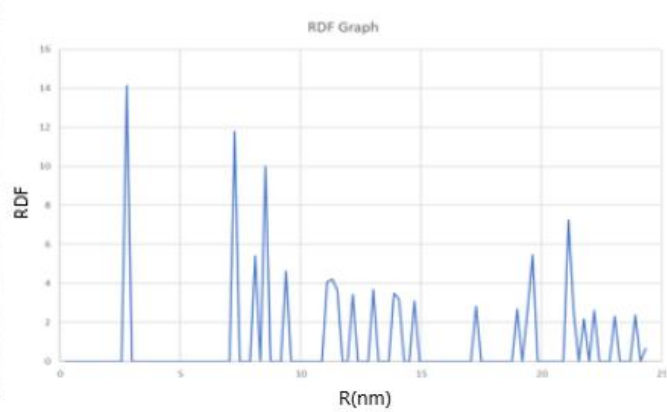

$\mathrm{R}(\mathrm{nm})$

Figure 2. (a) High-resolution image of $\square \square \square 2$ fabricated by hydrolysis of ceric ammonium nitrate; (b) Image and $\mathrm{RDF}$ of graphene liquid cell with $1.1 \mathrm{mM}$ concentration of $\mathrm{NaCl}$ with (-) $\mathrm{AuNP}$

\section{References}

[1] J.R.Jokisaari et al., Advanced Materials, 2018. 30(36), 1802702 (2018)

[2] C. Wang, T. Shokuhfar, and R.F. Klie, Advanced Materials, 2016. 28(35): p. 771

[3] L. Bhatt et al., Journal of Inorganic Biochemistry, 2020. 110997, ISSN 0162-0134

[4] N. de Jonge, and F. M. Ross. Nature Nanotechnology, 6, 695-704 (2011)

[5] J.M.Yuk et al., Science, 2012. p:61-64

[6] J.R. Jokisaari et al., ACS Nano, 201913 (3), 3151-3161

[7] This work is supported in part by the UIC Chancellor's Undergraduate Research Award. This work made use of Electron Microscopy Services at Research Resources Center, UIC 